\title{
INCIDENCIA DE LOS JUEGOS COOPERATIVOS EN LAS RELACIONES INTERPERSONALES
}

\author{
INCIDÊNCIA DOS JOGOS COOPERATIVOS NAS RELAÇÕES INTERPESSOAIS
}

INFLUENCE OF COOPERATIVE GAMES ON INTERPERSONAL RELATIONS

Juan Andueza*, Pere Lavega*

\begin{abstract}
Palabras clave: Educación Física. Investigaciónacción. Aceptación. Integración escolar.

Resumen: Este estudio tuvo como objetivo identificar los efectos de una unidad programática (UP) de juegos cooperativos, sobre díadas (aceptación y rechazo) y las características del grupo. Se siguió una metodología de investigación-acción; participaron 78 alumnos de 7 a 9 años $(M=8,3 ; D P=0,33), 38$ chicos $(48,7 \%)$ y 40 chicas $(51,3 \%)$. Al inicio y al final de la UP se aplicó un cuestionario sociométrico para identificar díadas reales de elección y de rechazo, y las dinámica grupales (Índice de Cohesión Grupal: ICG y del Índice de Disociación Grupal: IDG). Los resultados mostraron que los juegos cooperativos proporcionaron un aumento de díadas de aceptación, especialmente en chicas, y de rechazo en chicos; aumentó el ICG y el IDG; disminuyó el número de alumnos aislados. Este programa puede ser útil para fortalecer la dinámica intragrupo y para compactar el grupo.
\end{abstract}

Palavras chave: Educação Física. Pesquisa-ação. Aceitação. Integração escolar.

Keywords: Physical education. Action research. Acceptance. Integration.

Resumo: Este estudo teve como objetivo identificar os efeitos de uma unidade programática (UP) de jogos cooperativos sobre díades (aceitação e rejeição) e as características do grupo. A metodologia foi a pesquisa-ação; participaram 78 alunos de 7-9 anos $(M=8,3, D P=0,33), 38$ meninos $(48,7 \%)$ e 40 meninas $(51,3 \%)$. No início e no fim da UP foi aplicado um questionário sociométrico para identificar díades reais de escolha e rejeição e as dinâmicas de grupo (Índice de coesão do grupo: ICG e 0 Índice de dissociação do grupo: IDG). Os resultados mostraram que os jogos cooperativos proporcionaram um aumento de díades de aceitação, especialmente em mulheres, e rejeição nos homens; também aumentou o ICG e IDG e diminuiu o número de estudantes isolados. Este programa pode ser útil para fortalecer a dinâmica intragrupo e compactar o grupo.

\footnotetext{
Abstract: This study aims to identify the effects of a program unit (PU) based on cooperation motor tasks on dyads (acceptance and rejection) and group properties. It used action research methodology; participants were 78 7-9-years-old students $(M=8.3$, $S D=.33) ; 38$ boys ( $48.7 \%)$ and 40 girls $(51.3 \%)$. A sociometric questionnaire was applied at the beginning and at the end of the PU in order to identify real dyads of acceptation and rejection and group dynamics (Group Cohesion Index: GCl; group and group dissociation Index: GDI). The results showed that cooperative games increased acceptance dyads, especially in girls, and rejection dyads in boys. Both indexes increased while the number of isolated students decreased. This program can be useful to improve intragroup dynamics and for making the group compact.
}

*Universidad de Lleida. Lleida, España. E-mail: jandueza@xtec.cat

Recebido em: 25-05-2016 Aprovado em: 29-11-2016

(c) (i) () Licence 


\section{INTRODUCCIÓN}

La Educación Física (EF) es un medio idóneo para el desarrollo de la personalidad del alumno y de su proceso de socialización (ESPAÑA, 2014). Durante las clases, el maestro de EF dispone de un magnífico escenario para incentivar las relaciones entre el alumnado, evitar las situaciones discriminatorias, integrar a las personas aisladas y, en consecuencia, mejorar la convivencia y la cohesión grupal.

\subsection{La gestión de las relaciones del grupo de clase en EF}

La educación de las relaciones interpersonales exige al maestro el conocimiento exhaustivo de la dinámica de relaciones del grupo-clase. El cuestionario sociométrico es una herramienta útil y rápida para identificar las relaciones o díadas establecidas entre los alumnos (MORENO, 1972). La noción de díada se refiere a la relación entre dos alumnos que puede mostrarse en tres manifestaciones: la elección o aceptación, el rechazo y la indiferencia (PARLEBAS, 1992).

En el contexto del sistema educativo español, uno de los retos educativos del maestro es optimizar la dinámica de relaciones del grupo-clase para reducir el número de alumnos aislados, mejorar la cohesión grupal, minimizar los conflictos y las situaciones discriminatorias bien sean, por razón de género, cultura o discapacidad (ESPAÑA, 2014). Para abordar este desafío pedagógico, el maestro debe tomar decisiones estratégicas sobre las tareas a realizar en el aula y la forma de distribuir al alumnado en función de sus preferencias de elección y rechazo. Estas decisiones incidirán en la dinámica de relaciones del grupo-clase (ANDUEZA, 2015).

Atendiendo a las relaciones diádicas de aceptación, aquellas en las que los alumnos se eligen mutuamente, una primera decisión clave a adoptar por el docente implica la reducción del número de alumnos aislados, no integrados en los subgrupos. Algunos autores indican que el sentimiento de aceptación recíproca entre los alumnos otorga una mayor seguridad en el desarrollo de la personalidad, infundiendo respeto mutuo, y motivación por el aprendizaje, así como protección contra el estrés (DÍAZ-AGUADO; MARTIN, 2011). Así, el maestro puede servirse de los datos del cuestionario sociométrico, prestando atención sobre las relaciones recíprocas de aceptación real denominadas díadas de elección real (DER). Con estas díadas se podrá identificar los integrantes de los subgrupos y los alumnos aislados; valorar el nivel de consistencia de los subgrupos y determinar el Índice de Cohesión del grupo-clase (ICG). Consideramos que la incorporación de los alumnos aislados en diferentes subgrupos de clase puede ser una estrategia válida para favorecer su integración.

La segunda decisión atañe a la formación de subgrupos bien sean homogéneos 0 heterogéneos. Cuando la agrupación es libre, los alumnos tienden a participar con aquellos compañeros que comparten características comunes de género, marco cultural 0 nivel de competencia (GARCÍA-BACETE, 2006). Una vez forman parte de un subgrupo, se observan relaciones con diferente grado de consistencia según el tipo de DER. La mayor consistencia se detecta en la $D E R$ completa (dos alumnos se eligen y perciben ser elegidos recíprocamente), seguida por la DER incompleta (dos alumnos se eligen recíprocamente, y sólo uno percibe 
ser elegido por el otro); en cambio la mayor vulnerabilidad aparece en la DER simple (dos alumnos se eligen recíprocamente) (ANDUEZA, 2015). Algunos autores indican que los grupos homogéneos producen conductas prosociales entre los compañeros de subgrupos y reducen las conductas conflictivas con el resto del alumnado (BARSADE et al., 2000). Entendemos que el maestro debe actuar sobre las $D E R$ simples para que ganen en consistencia transformándose en DER completas.

La tercera decisión precisa tener una perspectiva global de la dinámica relacional del grupo-clase. Un indicador muy utilizado por la sociometría es el Índice de Cohesión del grupoclase (ICG). Este índice muestra el grado de cohesión del grupo. Entendemos por cohesión la capacidad del grupo de acumular el mayor número de díadas de aceptación. Así este índice se obtiene a partir del cociente entre el sumatorio de todas las DER y el número de posibles díadas. El incremento del ICG está asociado al mantenimiento de las DER ya existentes y a la integración de los alumnos aislados o las nuevas conexiones con otros compañeros de otros subgrupos. Desde esta perspectiva, consideramos que el número de subgrupos y el de integrantes de las tareas, así como el cambio de compañeros de subgrupo, resultan decisivos si se pretende fomentar la interacción entre todos los alumnos de la clase. Una buena estrategia para la integración de los alumnos aislados, consistiría en disponerlos en diferentes subgrupos junto a otros alumnos que comparten DER completas.

Respecto a las relaciones diádicas de rechazo, aquellas en las que los alumnos se rechazan mutuamente, identificamos dos decisiones clave; la primera debería llevar al docente a identificar los focos de conflicto que protagonizan los alumnos que son rechazados. Algunos autores han observado que los alumnos tienden a rechazar a los compañeros de diferente género, marco cultural o con intereses distantes (GARCÍA-BACETE, 2006). Para resolver este tipo de conflictos de manera pacífica (ESPAÑA, 2014) el maestro puede servirse de estrategias de distribución de los alumnos. La agrupación segregada de los alumnos por género tiende a fortalecer el crecimiento de conductas discriminatorias o sexistas, mientras que la agrupación mixta puede disminuir ese tipo de conductas (RAMOS; HERNÁNDEZ-MENDO, 2014; SIEDENTOP; HASTIE; VAN-DER-MARS, 2004). Por ello, optamos por buscar estrategias didácticas de agrupación mixta que potencien: la igualdad real de oportunidades entre chicos y chicas; el aumento de la participación de las chicas; la mejora de la percepción del otro género; y una actitud contraria a los estereotipos sexistas que comporte la eliminación de las conductas discriminatorias (BAENA; RUIZ, 2009; DÍAZ-AGUADO; MARTIN, 2011).

La segunda decisión referida al rechazo exige una intervención inmediata sobre un conflicto que perdura entre varios alumnos, en ocasiones asociados a conductas agresivas de acoso (OLWEUS, 1993). La ausencia de intervención sobre las conductas violentas facilita situaciones de acoso escolar con desagradables consecuencias (CEREZO; ATO, 2010; RIGBY, 2003). La normativa docente exige una inmediata actuación ante cualquier situación de acoso (ESPAÑA, 2014). En estas situaciones, conviene identificar las relaciones recíprocas de rechazo real denominadas díadas de rechazo real (DRR). Estas díadas permiten detectar la acumulación de rechazo recíproco o fricción entre individuos o grupos. Finalmente, el sumatorio de las díadas de rechazo por cada grupo-clase ofrece una visión de conjunto mediante el Índice de Disociación del grupo-clase (IDG). Entendemos por disociación la capacidad del grupo de acumular el mayor número de díadas de rechazo. 


\subsection{La elección de tareas en educación física}

Desde la teoría de la acción motriz (PARLEBAS, 2001) se considera que el maestro dispone de cuatro grandes grupos de situaciones motrices en función del tipo de relación (psicomotriz, cooperación, oposición y cooperación-oposición) que se origina entre los participantes. diversos autores reconocen la contribución de la cooperación para la integración de los alumnos con más dificultades, el desarrollo de conductas prosociales entre los alumnos y la cohesión del grupo (GARAIGORDOBIL, 2012; PUJOLÀS; LAGO; NARANJO, 2013; SUREDA; GARCÍA-BACETE; MONJAS, 2009; VELÁZQUEZ; FRAILE; LÓPEZ, 2014). LOS alumnos que cooperan se sienten más libres para actuar al producirse interacciones cara a cara, mostrando conductas abiertas, sin engaños que generan interdependencia, confianza mutua y amistad entre los participantes (GARAIGORDOBIL; FAGOAGA, 2006).

\subsection{Las Diferencias de Género en el Tipo de Tareas}

La intervención en las tareas motrices cooperativas demanda que el maestro preste especial atención al tipo de orientación de la tarea y a la presencia o ausencia de competición, para tratar de forma adecuada las diferencias de género con el fin de velar por la igualdad de oportunidades. Desde un punto de vista motivacional se han observado diferencias entre géneros; los chicos prefieren actividades orientadas al ego (comparar resultados y competir) mientras que las chicas valoran más las actividades orientadas a la consecución de una tarea (AMES, 1992; MORENO-MURCIA, et al., 2005).

Se ha observado que los juegos cooperativos sin competición, más practicados por el género femenino, pueden promover emociones positivas intensas (LAVEGA, et al., 2014), y resultan de mayor interés para favorecer la relación entre alumnos que el trabajo individual o el competitivo (ARITZETA; BALLUERKA, 2006)"container-title":"Psicothema","page":"757765","volume":"'18","issue":"'","source":"Google Scholar","abstract":"Cooperación, competición e interdependencia de objetivos en los equipos de trabajo: un enfoque mulln-Intinivel.InEste trabajo trata de predecir estilos de manejo de conflictos cooperativos y competitivos en 261 nequipos de trabajo de reciente construcción (tiempo 1, más practicado por el género masculino. En estas actividades, los alumnos deben establecer pactos con los compañeros, respetar las decisiones de los demás y adoptar estrategias comunes para conseguir un objetivo común (LAVEGA, 2006).

En cambio, el uso de la competición se ha asociado a la agresividad, más presente en los chicos, en la ejecución de las tareas (COLLARD, 2005; DUGAS, 2008). La distinción entre perdedores y ganadores comporta un sentimiento de discriminación o inferioridad en las chicas (MORENO-MURCIA, et al., 2005) y algunos chicos evidencien una falta de tolerancia hacia ellas por su menor nivel de competencia (ABBECASSIS, et al, 2002). En este sentido, las chicas tienden a ser más tolerantes que los chicos (GARAIGORDOBIL, 2009, 2012; GARAIGORDOBIL; GARCÍA-DE-GALDEANO, 2006).

Las tareas además de presentar diferentes tipos de interacción motriz y realizarse con 0 sin competición, también pueden implicar una relación motriz con o sin contacto corporal. Este último aspecto se ha relacionado con diferentes grados de violencia en función de la distancia que separa la interacción motriz entre los participantes (PARLEBAS, 2001). Estos aspectos 
internos de las tareas motrices unidos a otros factores de carácter subjetivo (amistades dentro de un grupo, preferencia o desagrado del contacto corporal) pueden favorecer las relaciones de amistad o estimular el rechazo cuando esas personas no comparten los mismos intereses, género o marco cultural.

A partir del marco teórico descrito, este estudio se planteó el objetivo de examinar el efecto de un programa de EF, basado en juegos de cooperación y estrategias didácticas integradoras, sobre la dinámica del grupo (referida a díadas de elección y de rechazo; índices de cohesión y de disociación grupal- ICG e IDG-; alumnos integrados y aislados; díadas intragrupales, intergrupales, en relación con los aislados y entre aislados).

\section{MÉTODO}

El presente estudio siguió una metodología de investigación-acción basada en cuatro fases: planificación (secuenciación de las unidades de programación), actuación (implementación de los juegos y distribución del alumnado), observación (recogida de opiniones de los alumnos registradas en el cuestionario sociométrico) y reflexión (valoración de los efectos de las tareas sobre la relaciones interpersonales y el comportamiento de los alumnos al participar en distintos grupos) (LATORRE, 2003). Se desarrolló como un plan piloto integrado en la Programación Anual de una escuela pública de Lleida, (España) ciudad interior con alto nivel de inmigración, con el objetivo de mejorar la cohesión social. La intervención obtuvo el consentimiento de los padres y la aprobación en el Consejo Escolar.

El maestro intervino como un investigador participante, compaginando la doble función de educador e investigador, sin afectar en las designaciones del alumnado y optimizando la dinámica del grupo a través de la selección de las tareas y la distribución de los alumnos. El alumnado durante todo el programa ofreció información al maestro sobre sus relaciones, comprobando la predisposición del maestro a modificar las agrupaciones para evitar los conflictos y fortalecer las amistades. Debido a ello, la reacción del alumnado al programa fue muy positiva, sin mostrar rechazo ni resistencia a las tareas.

\subsection{Participantes}

La muestra estuvo constituida por 78 alumnos de tercer nivel de la educación básica, repartidos en tres grupos de clase. La relación inicial entre los alumnos se caracterizó por diferentes niveles de cohesión en cada grupo (mayor, media menor), rango de edad de 8 a 9 años, 38 chicos (48'7\%) y 40 chicas (51'3\%), 25\% de alumnos de familias de inmigrantes y nivel sociocultural medio-bajo. Los criterios que se utilizaron para la selección de la muestra fueron: los grupos disponibles por el docente, la paridad de género y la edad de los alumnos de tercero de primaria, por tratarse de un momento de inicio de la asimilación y acomodación a las reglas, y de desarrollo de la perspectiva de los demás (PIAGET, 1996).

\subsection{Instrumento}

Con el fin de evaluar el objetivo propuesto, se utilizó el cuestionario sociométrico como instrumento útil y ágil para el estudio de las relaciones interpersonales (CORNEJO, 2006). 
El pasaje del cuestionario requirió la cumplimentación de las siguientes cuatro preguntas: a) ¿Con qué tres compañeros de clase prefieres jugar?; b) ¿Con qué tres compañeros de clase prefieres no jugar?; c) ¿Qué tres compañeros de clase crees que prefieren jugar contigo? y d) ¿Qué tres compañeros de clase crees que prefieren no jugar contigo?

La cumplimentación del cuestionario cumplió las siguientes normas de validez (CORNEJO, 2006). En primer lugar, se estableció un número fijo de designaciones elección (3) y de rechazo (3) para garantizar la igualdad de oportunidades, asumiendo el riesgo de una pérdida de expansividad (PARLEBAS, 1992). En segundo lugar, se comprobó la ausencia de autodesignación y la no repetición de los resultados entre las preguntas antagónicas (1-2; 3-4). En tercer lugar, se mantuvo el anonimato mediante la codificación de los nombres. Finalmente, se comprobó la validez del cuestionario mediante la prueba de pares, a partir de los datos obtenidos en el pretest y en el postest en cada uno de los tres grupos de estudio, obteniendo una correlación de .78, .80, .76.

\subsection{Procedimiento}

El presente estudio formó parte la cuarta unidad de una programación anual de EF compuesta por 9 unidades, situada entre los meses de enero y febrero, dedicada a los juegos de cooperación. Durante el mes de intervención se destacaron tres momentos clave: 1) pasaje y análisis del cuestionario sociométrico; 2) diseño y acomodación de las tareas; 3) intervención sobre la distribución de los alumnos.

Pasaje del cuestionario. El cuestionario sociométrico se aplicó antes y después del programa de cooperación, con las condiciones anteriormente descritas. Los resultados de cada test se pasaron a una matriz de transición (PARLEBAS, 1992), de donde se identificaron las díadas reales de elección y de rechazo. Finalmente, cada díada se representó gráficamente.

Diseño y acomodación de las tareas. El programa de cooperación comprendió ocho sesiones de una hora de duración que incluyeron 56 tareas (6-8 tareas por sesión) de cooperación sin competición. La distribución de las tareas siguió tres siguientes criterios: número de alumnos creciente, materiales variados, ausencia y presencia de contacto corporal (Ver Figura 1).

Figura 1 - Descripción y ordenación de las tareas del programa de cooperación

\begin{tabular}{|c|c|c|c|c|c|}
\hline Sesión & $\begin{array}{c}\text { № } \\
\text { juegos }\end{array}$ & $\begin{array}{c}\text { № personas } \\
\text { por juego }\end{array}$ & Material & $\begin{array}{l}\text { Contacto } \\
\text { corporal }\end{array}$ & Ejemplos \\
\hline 1 & 8 & $\begin{array}{l}\text { Parejas } \\
\text { Cuartetos } \\
\text { Gran grupo }\end{array}$ & $\begin{array}{l}\text { Globos } \\
\text { Pelotas } \\
\text { Sin }\end{array}$ & $\begin{array}{l}\operatorname{Sin} \\
\operatorname{Sin} \\
\text { Con }\end{array}$ & $\begin{array}{l}\text { Pasarse el globo sin que toque el suelo } \\
\text { Pasarse la pelota sin que toque el suelo } \\
\text { En fila, agarrados del compañero, sincronizar } \\
\text { los desplazamientos que inicia el primero }\end{array}$ \\
\hline 2 & 6 & $\begin{array}{l}\text { Parejas } \\
\text { Gran grupo }\end{array}$ & $\begin{array}{l}\text { Música } \\
\text { Música }\end{array}$ & $\begin{array}{l}\text { Con } \\
\text { Con }\end{array}$ & $\begin{array}{l}\text { Chocar las manos de la pareja según el ritmo } \\
\text { Realizar una coreografía grupal en la que } \\
\text { todos choquen las manos con los demás }\end{array}$ \\
\hline 3 & 6 & $\begin{array}{l}\text { Parejas } \\
\text { Cuartetos } \\
\text { Gran grupo }\end{array}$ & $\begin{array}{l}\text { Sin } \\
\text { Sin } \\
\text { Colchonetas }\end{array}$ & $\begin{array}{l}\text { Con } \\
\text { Con } \\
\text { Con }\end{array}$ & $\begin{array}{l}\text { Transportar al compañero subido a la espalda } \\
\text { Transportar a un compañero agarrándole de } \\
\text { las manos y los pies } \\
\text { Transportar al compañero sobre una } \\
\text { colchoneta entre más de seis alumnos }\end{array}$ \\
\hline
\end{tabular}




\begin{tabular}{|c|c|c|c|c|c|}
\hline \multirow{3}{*}{4} & \multirow{3}{*}{6} & Parejas & Pelotas & $\operatorname{Sin}$ & $\begin{array}{l}\text { Pasar al compañero la pelota de maneras } \\
\text { variadas }\end{array}$ \\
\hline & & Cuartetos & Pelotas & Con & $\begin{array}{l}\text { Sujetar una pelota con una parte del cuerpo y } \\
\text { agarrado a los compañeros }\end{array}$ \\
\hline & & Gran grupo & Pelotas & $\operatorname{Sin}$ & $\begin{array}{l}\text { Lanzar las pelotas para que choquen entre } \\
\text { todos }\end{array}$ \\
\hline \multirow{3}{*}{5} & \multirow{3}{*}{7} & Parejas, & Sin & Con & Representar objetos con el cuerpo \\
\hline & & Cuartetos & $\operatorname{Sin}$ & Con & Formar letras contactando con los compañeros \\
\hline & & Gran grupo & $\begin{array}{l}\text { Cuerdas y } \\
\text { aros }\end{array}$ & $\operatorname{Sin}$ & $\begin{array}{l}\text { Representar máquinas con cuerdas y aros } \\
\text { entre todos los compañeros }\end{array}$ \\
\hline \multirow{2}{*}{6} & \multirow{2}{*}{7} & Cuartetos & $\begin{array}{l}\text { Colchonetas } \\
\text { y pelotas }\end{array}$ & $\operatorname{Sin}$ & $\begin{array}{l}\text { Transportar pelotas sobre una colchonetas sin } \\
\text { que caigan al suelo (en grupos de 4) }\end{array}$ \\
\hline & & Gran grupo & Cuerdas & $\operatorname{Sin}$ & $\begin{array}{l}\text { Situados en fila (8 alumnos), saltar la cuerda } \\
\text { siguiendo un orden }\end{array}$ \\
\hline \multirow{3}{*}{7} & \multirow{3}{*}{6} & Parejas, & Aros & $\operatorname{Sin}$ & Intercambiar el aro con el compañero \\
\hline & & Cuartetos & Picas & $\operatorname{Sin}$ & Entre cuatro, mantener la pica, soltarla y \\
\hline & & Gran grupo & Aros & $\operatorname{Sin}$ & $\begin{array}{l}\text { Pasar por dentro de la fila de aros, cada } \\
\text { alumno aguanta un aro }\end{array}$ \\
\hline \multirow{2}{*}{8} & \multirow{2}{*}{8} & & $\begin{array}{l}\text { Periódicos y } \\
\text { música }\end{array}$ & Con & $\begin{array}{l}\text { Bailar agarrado del compañero, y al detener la } \\
\text { música bailar encima de hojas de periódico }\end{array}$ \\
\hline & & Gran grupo & $\begin{array}{l}\text { Sillas y } \\
\text { Música }\end{array}$ & Con & $\begin{array}{l}\text { Bailar libremente, y al detener la música } \\
\text { sentarse en las sillas }\end{array}$ \\
\hline
\end{tabular}

Fuente: Datos de los autores

Intervención sobre la distribución de los alumnos. La representación gráfica sirvió para establecer unas primeras agrupaciones de partida. La primera intervención consistió en la aplicación de estrategias de integración de los alumnos aislados. En segunda instancia cuando los grupos eran más numerosos, se adoptó una estrategia de agrupación mixta obligatoria. Por último, se incentivó la interacción entre todos los alumnos.

\subsection{Análisis de los datos}

El análisis de los datos obtenidos en el cuestionario sociométrico generó información sobre las díadas y la dinámica grupal. La perspectiva diádica permitió conocer la evolución de las díadas de elección y de rechazo de forma cualitativa (completas, incompletas y simples) y cuantitativa (incremento, mantenimiento o descenso).

La perspectiva grupal ofreció una visión global del grupo clase a través del nivel de cohesión-ICG- y del grado de disociación -IDG-; las propiedades de los grupitos (conexión en cadena, densidad, vulnerabilidad, compactación) y la dinámica de relaciones (intragrupal, intergrupal, con los aislados y entre los aislados).

\section{RESULTADOS}

Los resultados de esta investigación se centran en la comparación de los datos obtenidos mediante el cuestionario sociométrico en dos momentos del programa de intervención (antes y después). Los datos se presentan desde dos perspectivas: efectos sobre las díadas y efectos sobre la dinámica grupal. 


\subsection{Efectos sobre las díadas}

El cuestionario sociométrico aportó tres informaciones relevantes sobre los efectos en las díadas:

- La cuantificación de díadas de elección y rechazo, diferenciando díadas reales y percibidas. En lo que se refiere a las elecciones, el programa de cooperación comportó un incremento notable de las díadas reales (E.Real), y un descenso de las díadas percibidas (E.Percibida). Por otra parte, se produjo un mayor aumento en las diadas de rechazo real (R.Real) y percibido (R.Percibido), especialmente en los grupos menos cohesionados (G2-G3) (Figura 2).

Figura 2 - Evolución del número de díadas reales y percibidas de elección y de rechazo

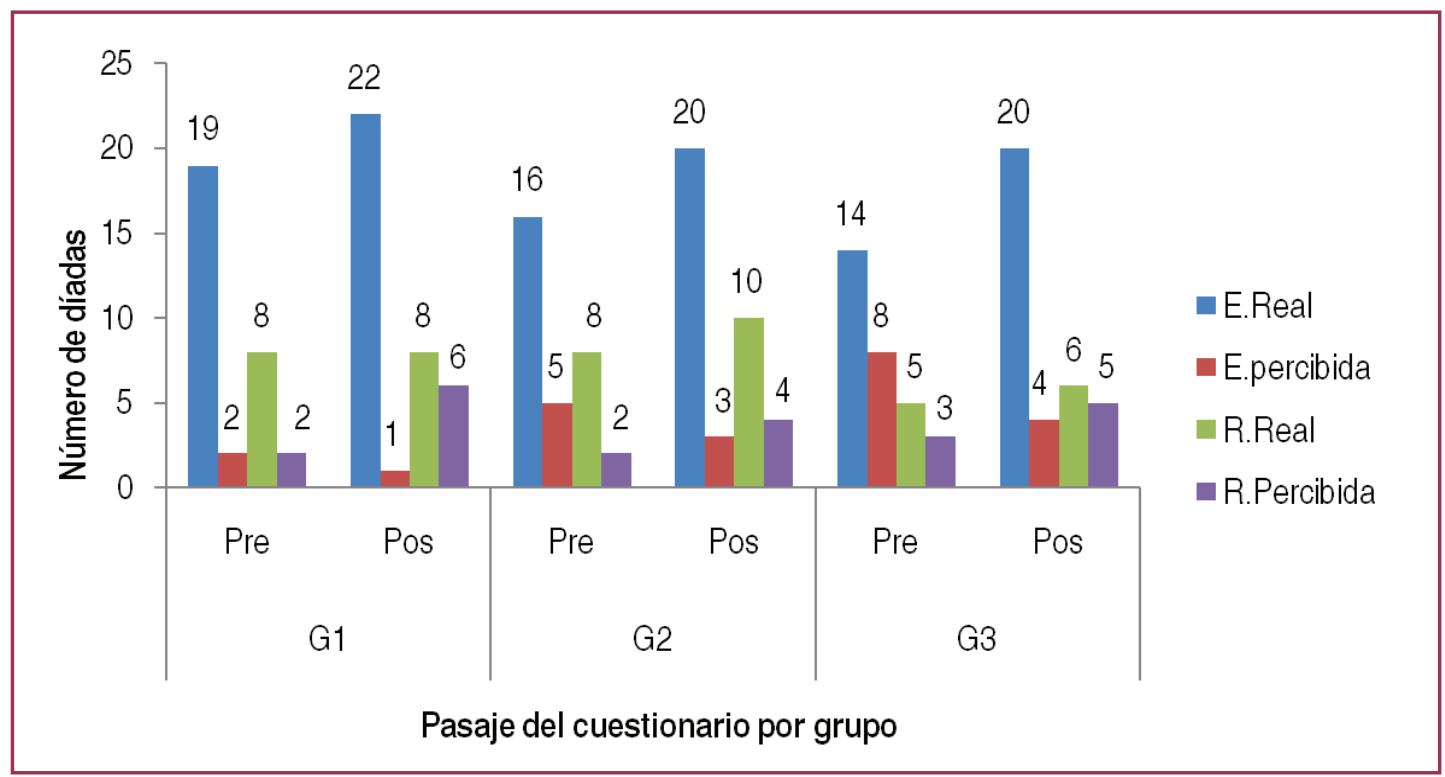

Fuente: Datos de los autores

- La evolución cualitativa de los tipos de díadas. El programa de cooperación mejoró la calidad de las relaciones al elevarse el número de las díadas de elección completas y reducirse las diadas de rechazo completas (Tabla 1).

Tabla 1 - Evolución del tipo de díadas de elección y de rechazo real

\begin{tabular}{cccccccccc}
\hline TIPO DE DÍADAS & & G1 & & & G2 & & \multicolumn{2}{c}{ G3 } \\
\hline Elección real & Pre & Pos & Dif & Pre & Pos & Dif & Pre & Pos & Dif \\
\hline Completas & 17 & 19 & 2 & 11 & 11 & 0 & 10 & 15 & 5 \\
Incompletas & 1 & 3 & 2 & 4 & 7 & 3 & 2 & 4 & 2 \\
Simples & 1 & 0 & -1 & 1 & 2 & 1 & 2 & 1 & -1 \\
\hline Rechazo real & & & & & & & & & \\
\hline Completas & 4 & 4 & 0 & 2 & 1 & -1 & 1 & 0 & -1 \\
Incompletas & 3 & 4 & 1 & 4 & 3 & -1 & 2 & 5 & 3 \\
Simples & 1 & 0 & -1 & 2 & 6 & 4 & 2 & 1 & -1 \\
\hline
\end{tabular}

Nota: G1=grupo cohesión mayor; G2=grupo cohesión media; G3=grupo cohesión menor; Pre=Pretest; Pos=Postest; Dif=Diferencia; 
- La evolución de las relaciones desde la perspectiva de género. Se observó un incremento de elecciones reales mayor en las chicas que en los chicos. En cambio, el incremento del rechazo real se localizó en la relación intergénero, especialmente en los grupos menos cohesionados (Tabla 2).

Tabla 2 - Evolución del número de díadas de elección y rechazo real según género

\begin{tabular}{cccccccccc}
\hline GÉNERO & & G1 & \multicolumn{3}{c}{ G2 } & \multicolumn{3}{c}{ G3 } \\
\hline Elección real & Pre & Pos & Dif & Pre & Pos & Dif & Pre & Pos & Dif \\
\hline Mixto & 0 & 0 & 0 & 4 & 5 & 1 & 1 & 0 & -1 \\
Chicos & 10 & 11 & 1 & 6 & 7 & 1 & 6 & 9 & 3 \\
Chicas & 9 & 11 & 2 & 6 & 8 & 2 & 7 & 11 & 4 \\
\hline Rechazo real & & & & & & & & & \\
\hline Mixto & 7 & 6 & -1 & 5 & 8 & 3 & 2 & 3 & 1 \\
Chicos & 0 & 1 & 1 & 2 & 1 & -1 & 3 & 3 & 0 \\
Chicas & 1 & 1 & 0 & 1 & 1 & 0 & 0 & 0 & 0 \\
\hline
\end{tabular}

Nota: G1=grupo cohesión mayor; G2=grupo cohesión media; G3=grupo cohesión menor; Pre=Pretest; Pos=Postest; Dif=Diferencia; Fuente: Datos de los autores

\subsection{Efectos sobre la dinámica grupal}

La valoración global de los efectos del programa sobre el grupo de clase obtenido mediante los índices de cohesión grupal (ICG) y de disociación grupal (IDG) reflejó una evolución relacionada con el nivel de cohesión de cada grupo. Así, se obtuvo un ascenso del nivel de ICG y un descenso del IDG en los grupos menos cohesionados (Figura 3).

Figura 3 - Evolución del ICG y del IDG

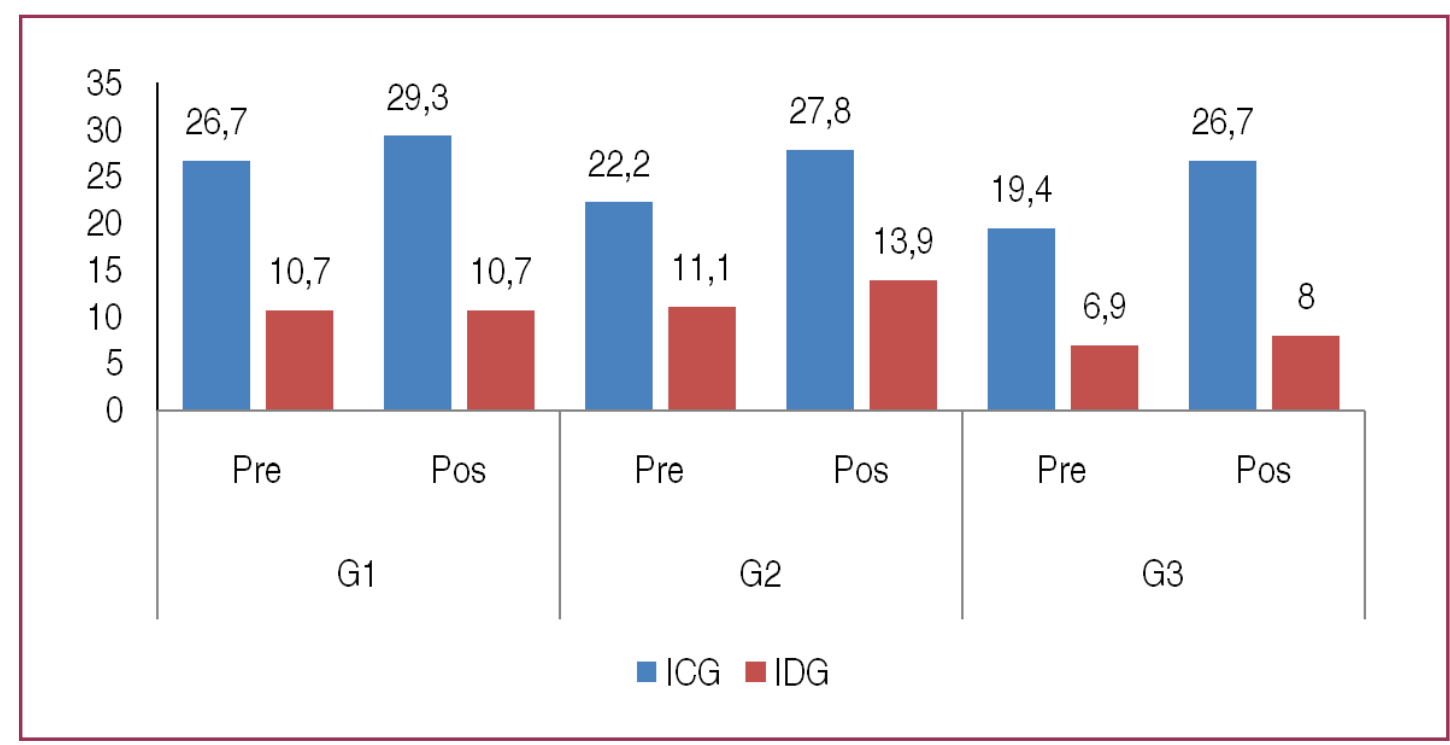

Nota: G1=grupo cohesión mayor; G2=grupo cohesión media; G3=grupo cohesión menor; Pre=Pretest; Pos=Postest;

Fuente: Datos de los autores 
La valoración de la dinámica de los subgrupos se realizó a partir de la identificación de los alumnos integrados y los alumnos aislados (con y sin díadas reales de elección, respectivamente). El programa de cooperación facilitó la incorporación de alumnos aislados en los subgrupos, siendo mayor en los grupos menos cohesionados (G2G3), especialmente chicos (Tabla 4). Una vez identificados los alumnos integrados, la representación gráfica de las díadas de elección real del cuestionario sociométrico permitió reconocer el número de subgrupos, y las propiedades de los mismos. Este programa redujo el número de subgrupos en los grupos más cohesionados (G1-G2) y aumentó en el menos cohesionado (G3) (Tabla 3).

Tabla 3 - Evolución del número de alumnos aislados y de subgrupos

\begin{tabular}{cccccccccc}
\hline & \multicolumn{3}{c}{ G1 } & \multicolumn{3}{c}{ G2 } & \multicolumn{3}{c}{ G3 } \\
\hline Aislados & Pre & Pos & Dif & Pre & Pos & Dif & Pre & Pos & Dif \\
\hline Chicos & 3 & 3 & 0 & 4 & 2 & -2 & 4 & 1 & -3 \\
Chicas & 4 & 2 & -2 & 3 & 1 & -2 & 4 & 3 & -1 \\
\hline
\end{tabular}

\begin{tabular}{clllllllll} 
Subgrupos & & & & & & & & & \\
Número & 6 & 5 & -1 & 4 & 3 & -1 & 4 & 6 & 2 \\
\hline
\end{tabular}

Nota: G1=grupo cohesión mayor; G2=grupo cohesión media; G3=grupo cohesión menor; Pre=Pretest; Pos=Postest; Dif=Diferencia; Fuente: Datos de los autores

La representación gráfica desvela las propiedades de los subgrupos. Estas propiedades fueron cuantificadas a través del número de alumnos encadenados (conexión), así como el número de díadas de elección real (compactación, tres díadas; vulnerabilidad, una diada). El programa de cooperación comportó un aumento del número de integrantes y un alargamiento de la cadena en los grupos menos cohesionados (G2-G3), así como un incremento notable de la compactación (Tabla 4).

Tabla 4 - Evolución del número de las propiedades de los subgrupos

\begin{tabular}{cccccccccc}
\hline & \multicolumn{3}{c}{ G1 } & \multicolumn{3}{c}{ G2 } & \multicolumn{3}{c}{ G3 } \\
\hline Propiedades & Pre & Pos & Dif & Pre & Pos & Dif & Pre & Pos & Dif \\
\hline Conexión & 6 & 5 & -1 & 6 & 7 & 1 & 5 & 9 & 4 \\
grupo numeroso & 6 & 5 & -1 & 7 & 11 & 4 & 7 & 10 & 3 \\
Compactación & 7 & 9 & 2 & 4 & 6 & 2 & 4 & 6 & 2 \\
Vulnerabilidad & 7 & 5 & -2 & 8 & 10 & 2 & 10 & 10 & 0 \\
\hline
\end{tabular}

Nota: G1=grupo cohesión mayor; G2=grupo cohesión media; G3=grupo cohesión menor; Pre=Pretest; Pos=Postest; Dif=Diferencia; Fuente: Datos de los autores

Por último, la representación gráfica también establece una distinción de las díadas de elección y de rechazo en cuatro focos de interacción: intragrupal, intergrupal, con los aislados y entre los aislados. El programa de cooperación originó un aumento de las díadas intragrupales de elección, un descenso de las díadas con los aislados; y también un incremento del número de díadas de rechazo real intergrupal en los grupos menos cohesionados (Tabla 5). 
Tabla 5 - Evolución de las díadas de elección según el foco de interacción

\begin{tabular}{cccccccccc}
\hline & & G1 & \multicolumn{3}{c}{ G2 } & & G3 \\
\hline ELECCIÓN & Pre & Pos & Dif & Pre & Pos & Dif & Pre & Pos & Dif \\
\hline E.R. Intragrupal & 19 & 22 & 3 & 16 & 20 & 4 & 14 & 20 & 6 \\
\hline RECHAZO & & & & & & & & & \\
\hline R.R. Intragrupal & 0 & 0 & 0 & 1 & 1 & 0 & 0 & 0 & 0 \\
R.R. Intergrupal & 7 & 5 & -2 & 1 & 8 & 7 & 2 & 5 & 3 \\
R.R. Con aislados & 1 & 3 & 2 & 6 & 1 & -5 & 1 & 1 & 0 \\
R.R. Interaislados & 0 & 0 & 0 & 0 & 0 & 0 & 2 & 0 & -2 \\
\hline
\end{tabular}

Nota: G=grupo cohesión mayor; G2=grupo cohesión media; G3=grupo cohesión menor; Pre=Pretest; Pos=Postest; Dif=Diferencia; E.R=Elección real; R.R=Rechazo real. Fuente: Datos de los autores

\section{CONCLUSIONES Y DISCUSIÓN}

El objetivo de este estudio pretende observar los efectos de un programa de educación física, basado en tareas motrices de cooperación, sobre las relaciones entre los alumnos del grupo clase. Algunos autores orientan el estudio de las relaciones interpersonales en función de las preferencias de elección o rechazo de los compañeros de juego con la intención de favorecer al maestro el manejo de la distribución de los alumnos durante las tareas y la gestión de la dinámica del grupo de clase. Para este cometido, el maestro enmarca su intervención en un proceso de investigación-acción, concretando decisiones sobre la observación, las tareas a realizar y la distribución de los alumnos (ANDUEZA, 2015).

Para la observación de las preferencias en las relaciones el cuestionario sociométrico es un instrumento ágil y aplicable. Este recurso didáctico ofrece una gran variedad de posibilidades de adaptación en cuanto a las preguntas a contestar, el número de designaciones, los indicadores de observación y el sistema de representación (CORNEJO, 2006). La adaptación del instrumento exige la toma de una serie de decisiones por parte del maestro:

1. El maestro puede fomentar la espontaneidad (PARLEBAS, 1992), indicar un número apropiado de designaciones 0 incorporar el orden de preferencia (ARRUGA, 1983). Consideramos de gran utilidad la designación de tres alumnos para grupos numerosos, al facilitar la confección de grupos más estables y ayudar en la detección de puntos de conflicto.

2. Algunas investigaciones abordan el estudio de las relaciones buscando las diferencias individuales para identificar a los alumnos por su rol social de popular, rechazado, controvertido, normal e ignorado (GARCÍA-BACETE, 2006; SUREDA; GARCÍA-BACETE; MONJAS, 2009). Esta perspectiva no permite diferenciar con claridad a los alumnos aislados de los integrados. Por ello, planteamos un análisis de las díadas que aporte una información relevante a la hora de distribuir a los alumnos; en el momento de identificar a alumnos populares que no se sienten integrados en los subgrupos de clase y carecen de una relación recíproca; y cuando se observa a los alumnos que no son considerados como rechazados pero que presentan conflictos interpersonales y dificultan la dinámica del grupo de clase. 
3. A partir de los datos del cuestionario se pueden diferenciar 81 tipos de díadas que incluyen relaciones de elección, rechazo e indiferencia desde una perspectiva real y percibida (PARLEBAS, 1992). La selección de las díadas reales de elección y de rechazo permite destacar tres tipos de díadas (completas, incompletas o simples); también facilita la comprensión global del fenómeno mediante la representación gráfica en la que se identifican los subgrupos, sus propiedades (conexión en cadena, compactación, vulnerabilidad) y su dinámica (ANDUEZA, 2015).

Una vez obtenidos los datos del cuestionario, el maestro puede adaptar las tareas al grupo de clase fomentando el trabajo grupal sin discriminaciones por razón de género o capacidad (RD 126/2014). Para ello, el docente dispone de tareas con diferentes tipos de interacción motriz -psicomotriz, de cooperación, cooperación-oposición y oposición- (PARLEBAS, 2001). Algunos autores reconocen un mayor rendimiento de las tareas de cooperación sobre otros tipos de tareas caracterizadas por el individualismo, la oposición o la competición (DURAND, 2007). En la línea de lo que apuntan otros estudios, el programa de cooperación se considera un procedimiento capaz de incrementar la empatía, la ayuda, y la cohesión del grupo (e.g., LEÓN; GOZALO; POLO, 2012; SUREDA; GARCÍA-BACETE; MONJAS, 2009); generar efectos positivos sobre las relaciones de amistad entre los compañeros de clase y promover conductas prosociales que refuerzan la confianza mutua (GARAIGORDOBIL, 2012; GARAIGORDOBIL; FAGOAGA, 2006); Los hallazgos de este estudio coinciden con observaciones identificadas en otras investigaciones como que las tareas de cooperación: promueven las relaciones intragrupales dotando al grupo de mayor consistencia debido al incremento de díadas de elección completas. Esta dinámica facilita la integración de alumnos aislados, genera mayor interdependencia entre los miembros de cada subgrupo, y mejora la calidad de las relaciones gracias a un aumento de díadas completas (díadas real y percibida).

En cambio, si bien algunos autores afirman que las tareas cooperativas reducen los conflictos y la violencia entre los alumnos (e.g., LEÓN; GOZALO; POLO, 2012; SUREDA; GARCÍA-BACETE; MONJAS, 2009), en este estudio se ha observado un incremento del número de díadas reales de rechazo. Esta circunstancia podría deberse a la participación en tareas motrices con contacto corporal que solicitaban la interacción con personas del otro género.

\subsection{La estrategia de agrupación}

El maestro también debe considerar el incumplimiento de las reglas (conductas motrices perversas, (SÁEZ-DE-OCÁRIZ; LAVEGA, 2013) como un aspecto de especial relevancia para el control de la dinámica del grupo. En este sentido, la estrategia de cambio de grupo frente a las situaciones de conflictividad grupal, y el mantenimiento de estructuras grupales heterogéneas (formación de subgrupos numerosos mixtos e interculturales) generan tensiones entre las personas de diferente condición (género, marco cultural, capacidad motriz, etc).

\subsection{El género}

Los alumnos tienden a agruparse por afinidad cuando participan en tareas cooperativas (DURAND, 2007). La agrupación por afiliación suele favorecer las relaciones interpersonales entre alumnos del mismo género. Paralelamente el rechazo se suele producir en las relaciones 
entre ambos géneros. En el presente estudio, se incorporó una condición en las agrupaciones que garantizaba la coeducación. Considerando que los alumnos de primaria tienden a establecer sus relaciones entre compañeros del mismo género, los chicos en un lado y las chicas en otro, constatamos que la estrategia de agrupación mixta apenas generó cambios en las díadas de elección real intergénero.

Algunos autores han observado una conducta diferencial asociada al género. Las chicas suelen establecer relaciones de amistad de una a una, dándole un mayor valor a los sentimientos y a la intimidad; en tanto que los chicos participan de manera más colectiva reuniéndose en subgrupos, equipos o pandillas, en donde las relaciones se establecen entre un número impreciso de personas (BENENSON; CHRISTAKOS, 2003; ESTELL, et al., 2002; WEISS; SMITH, 2002). En nuestro estudio se observa un mayor incremento de las díadas de elección real entre chicas respecto a los chicos. Ello puede asociarse a que las chicas se muestran más empáticas (TAYLOR, et al., 2002) y utilizan estrategias más cooperativas (BUKOWSKI; SIPPOLA, 2002; WEISS; SMITH, 2002).

En resumen, consideramos que el conocimiento de la dinámica de relaciones del grupoclase, mediante cuestionarios sociométricos ágiles, unido a la intervención basada en tareas motrices cooperativas y sistemas de distribución-agrupación que contemplen la diversidad y la afiliación, son herramientas muy útiles para que el maestro pueda optimizar las relaciones de su grupo de clase.

\section{REFERENCIAS}

ABBECASSIS, Maurissa et al. Mutual antipathies and their significance in middle childhood and adolescence. Child Development, v. 73, n. 5, p.1543-1556, sept./oct. 2002.

AMES, Carole. Classrooms: goals, structures and student motivation. Journal of educational psychology, v. 84, n. 3, p. 261-271, sept. 1992.

ANDUEZA, Juan Antonio. Educar las relaciones interpersonales a través de la educación física en primaria. 2015. $611 \mathrm{f}$. Tesis (Doctoral). Lleida: INEFC-Lleida, Universidad de Lleida. 2015.

ARITZETA, Aitor; BALLUERKA, Nekane. Cooperation, competition and goal interdependence in work teams: A multilevel approach. Psicothema, v. 18, n. 4, p. 757-765, nov. 2006.

ARRUGA, Arthur. Introducción al test sociométrico. Barcelona: Herder, 1983.

BAENA, Antonio; RUIZ, Pedro Jesús. Tratamiento educativo de la coeducación y la igualdad de sexos en el contexto escolar y en especial en educación física. Aula Abierta, v. 37, n. 2, p. 111-122, 2009.

BARSADE, Sigal et al. To your heart's content: A model of affective diversity in top management teams. Administrative Science Quarterly, v. 45, n. 4, p. 802-836, dec. 2000.

BENENSON, Joyce F; CHRISTAKOS, Athena. The greater fragility of females' versus males' closest same-sex friendships. Child Development, v. 74, p. 1123-1129, jul./aug. 2003.

BUKOWSKI, William; SIPPOLA, Lorrie. K. Groups, individuals, and victimization: a view of the peer system. In: JUVONEN, Jaana; GRAHAM, Sandra (Ed.). Peer harassment in school: the plight of the vulnerable and victimized. New York: Guilford press, 2001. p. 355-357. 
CEREZO, Fuensanta; ATO, Manuel. Social status, gender, classroom climate and bullying among adolescents pupils. Anales de psicología, v. 26, n. 1, p. 137-144, jan. 2010.

COIE, John; DODGE, Kenneth. A; COPPOTELLI, Heide. Dimensions and types of social status: a cross-age perspective. Developmental Psychology, v. 18 n. 4, p. 557-570, jul. 1982.

COLLARD, Luc. Conflits et prises de risque en EPS. Carrefours de l'Éducation, Amiens, v. 20, n. 2 , p. 13-26, dec. 2005.

CORNEJO, José Manuel. El análisis de las interacciones grupales: las aplicaciones SOCIOS. Anuario de Psicología, v. 37, n. 3, p. 277-298, 2006.

DÍAZ-AGUADO, María José; MARTIN, Gema. Convivencia y aprendizaje escolar en la adolescencia desde una perspectiva de género. Psicothema, v. 23, n. 2, p. 252-259, abr. 2011.

DUGAS, Éric. Sport et effets éducatifs à l'école: de la violence à l'agressivité motrice. International Journal on Violence and School, n. 5, p. 67-83, 2008.

DURAND, Claire. La coprésence en éducation physique et sportive: les effets de quatre modalités relationnelles de pratique adoptées en kayak au cours moyen. Staps, v. 77, n. 3, p. 29-47, 2007.

ESPAÑA. Real decreto 126/2014, de 28 de febrero, por el que se establece el currículo básico de la Educación Primaria. Boletín Oficial del Estado de España, n. 52, p. 19354, 2016.

ESTELL, David et al. D. Social relations and academic achievement in inner-city early elementary classrooms. International Journal of Behavioral Development, v. 26, n. 6, p. 518-528, nov. 2002.

GARAIGORDOBIL, Maite. A comparative analysis of empathy in childhood and adolescence: Gender differences and associated socio-emotional variables. International Journal of Psychology and Psychological Therapy, v. 9, n. 2, p. 217-235, 2009.

GARAIGORDOBIL, Maite. Cooperative conflict-solving during adolescence: Relationships with cognitive behavioural and predictor variables. Infancia y Aprendizaje, v. 35, n. 2, p. 151-165, mayo 2012.

GARAIGORDOBIL, Maite; GARCÍA DE GALDEANO, Patricia. Empatía en niños de 10 a 12 años. Psicothema, v. 18, n. 2, p. 180-186, mayo 2006.

GARAIGORDOBIL, Maite; FAGOAGA, José María. El juego cooperativo para prevenir la violencia en los centros escolares: evaluación de programas de intervención para la educación infantil, primaria y secundaria. Madrid: Ministerio de Educación, 2006.

GARCÍA-BACETE, Francisco Juan. La identificación de los alumnos rechazados. Comparación de métodos sociométricos de nominaciones bidimensionales. Infancia y Aprendizaje, v. 29, n. 4, p. 437451, nov. 2006.

LATORRE, Antonio. La investigación-acción: conocer y cambiar la práctica educativa. Barcelona: Graó, 2003.

LAVEGA, Pere et al. Efecto de la cooperación motriz en la vivencia emocional positiva: perspectiva de género. Movimento, v. 20, n. 2, p. 593-618, abr./jun. 2014.

LEÓN, Benito; GOZALO, Margarita; POLO, María Isabel. Aprendizaje cooperativo y acoso entre iguales. Infancia y Aprendizaje, v. 35, n. 1, p. 23-35, feb. 2012. 
MORENO, Jakob Levi. Fundamentos de la Sociometría. Buenos Aires: Paidós, 1972.

MORENO-MURCIA, José Antonio et al. Motivación, disciplina, coeducación y estado de flow en educación física: Diferencias según la satisfacción, la práctica deportiva y la frecuencia de práctica.

Cuadernos de Psicología del Deporte, v. 5, n. 1-2, p. 231-243, 2005.

OLWEUS, Dan. Conductas de acoso y amenaza entre escolares. Madrid: Morata, 1998.

PARLEBAS, Pierre. Juegos, deportes y sociedades: léxico de praxiología motriz. Barcelona: Paidotribo, 2001.

PARLEBAS, Pierre. Sociométrie, réseaux et communication. Paris: PUF, 1992.

PIAGET, Jean. Psicología de la inteligencia. Buenos Aires: Psique, 1996.

PUJOLÀS, Pere; LAGO, José Ramón; NARANJO, Milagros. Aprendizaje cooperativo y apoyo a la mejora de las prácticas inclusivas. Revista de Investigación en Educación, v. 11, n. 3, p. 207-218, 2013.

RAMOS, Fernando; HERNÁNDEZ-MENDO, Antonio. Intervención para la reducción de la discriminación por sexo en las clases de educación física según los contenidos y agrupamientos utilizados. Revista Española de Educación Física y Deportes, n. 404, p. 27-38, ene. 2014.

RIGBY, Ken. Consequences of bullying in schools. The Canadian Journal of Psychiatry, n. 48, p. 583-590, oct. 2003.

SÁEZ-DE-OCÁRIZ, Unai; LAVEGA, Pere. Transformar conflictos en educación física en primaria a través del juego: aplicación del índice de conflictividad. Revista Cultura y Educación, v. 25, n. 4, p. 549-560, dic. 2013.

SIEDENTOP, Deryl; HASTIE, Peter; VAN-DER-MARS, Hans. Complete guide to sport education. Champaign: Human Kinetics, 2004.

SUREDA, Inmaculada; GARCÍA-BACETE, Francisco Juan; MONJAS, María Inés. Razones de niños y niñas de diez y once años para preferir o rechazar a sus iguales. Revista Latinoamericana de Psicología, v. 41, n. 2, p. 305-321, jul. 2009.

TAYLOR, Catherine et al. Gender differences in middle school adjustment, physical fighting, and social skills: evaluation of a social competency program. The Journal of Primary Prevention, v. 23, n. 2, p. 259-272, dec. 2002.

VELÁZQUEZ, Carlos; FRAILE, Antonio; LÓPEZ, Víctor M. Aprendizaje cooperativo en Educación Física. Movimento, v. 20, n. 1, p. 239-259, jan./mar. 2014.

WEISS, Maureen; SMITH, Alan. Friendship quality in youth sport: relationship to age, gender, and motivation variables. Journal of Sport and Exercise Psychology, v. 24, n. 4, p. 420-437, dec. 2002. 
